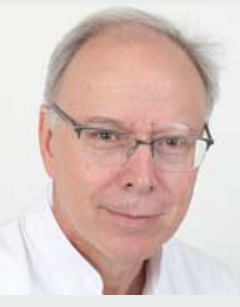

Prof. Dr. Christoph Josten

\section{Wirbelsäulenverletzungen im Kindesalter}

Verletzungen der kindlichen Wirbelsäule sind insgesamt selten und werden in der Literatur mit 1-4\% aller Frakturen bei Kindern und Jugendlichen angegeben.

Eine besondere Herausforderung stellt neben der adäquaten Erstversorgung eine zielgerichtete Diagnostik dar, denn Wirbelsäulenverletzungen im Kindesalter treten fast ausschließlich im Rahmen von Hochrasanztraumata auf. Hier sind vor allem Stürze aus großer Höhe sowie Verkehrsunfälle zu nennen, aber auch Misshandlungen im Kleinstkindesalter. Ab dem 6. Lebensjahr bis zum Jugendalter treten dann neben den Verkehrsunfällen auch zunehmend Sportunfälle in den Vordergrund.

Aufgrund des Unfallmechanismus sind Wirbelsäulenverletzungen im Kindes- und Jugendalter häufig assoziiert mit schwerwiegenden Begleitverletzungen, einer nicht zu unterschätzenden Inzidenz an neurologischen Komplikationen und einer hohen Mortalität, besonders bei Verletzungen und Instabilitäten der Halswirbelsäule.

Die kindlichen Wirbelsäulenverletzungen geben zudem kein einheitliches Bild ab. Sowohl die Verletzungsmuster, als auch die daraus resultierenden Therapieansätze unterscheiden sich Bezug auf die verschiedenen Altersklassen zum Teil erheblich.

Das Spektrum reicht von der „einfachen“ Orthesen- Ruhigstellung über die durchaus wertvolle Therapie mittels Halo-Fixateur bis hin zu aufwendigen Operationsverfahren, insbesondere in der okzipito-cervicalen Region.

Dementsprechend ist eine schnelle und zielgerichtete Diagnostik von essentieller Bedeutung für das Outcome, was eine genaue Kenntnis der altersspezifischen radiologischen Besonderheiten und der zu erwartenden Verletzungsmuster voraussetzt. Erschwerend kommt hinzu, dass im Kindesalter bei 19-34\% aller Rückenmarksverletzungen keine röntgenologischen Zeichen einer Fraktur oder Instabilität zu finden sind.

Anhand dieser Kenntnisse wird deutlich, dass die Behandlung von schwer Wirbelsäulen-verletzten Kindern, besonders bei begleitender Neurologie nicht nur für die Kinder selbst und deren Eltern eine große psychische und emotionale Belastung bedeutet, sondern auch die behandelnden Ärzte vor große diagnostische und therapeutische Herausforderungen stellt. Sicherlich gibt es kaum einen Bereich in der Frakturversorgung, bei dem eine derartig große Unsicherheit bezüglich der richtigen Diagnostik und Therapie besteht wie bei der kindlichen Wirbelsäule.

Deshalb wird es in dieser Ausgabe unserer Zeitschrift zum Ziel gemacht, praxisnah die Diagnostik und differenzierte Therapie der kindlichen Wirbelsäulenverletzung zu bearbeiten.

Ich wünsche Ihnen viel Freude und hoffentlich ebenso viel Erkenntnisgewinn beim Lesen dieser Ausgabe

Ihr

Christoph Josten 